\title{
Optical characterization of InGaAsN/GaAsN/GaAs quantum wells with InGaP cladding layers
}

\author{
C.R. Lu ${ }^{\text {a,* }}$, H.L. Liu ${ }^{a}$, J.R. Lee ${ }^{a}$, C.H. Wu ${ }^{a}$, H.H. Lin ${ }^{b}$, L.W. Sung ${ }^{b}$ \\ ${ }^{a}$ Department of Physics, National Taiwan Normal University, Taipei 116, Taiwan, ROC \\ ${ }^{\mathrm{b}}$ Institute of Electrical Engineering, National Taiwan University, Taipei 116, Taiwan, ROC
}

\begin{abstract}
Optical properties of InGaAsN/GaAs and InGaAsN/GaAsN/GaAs quantum well structures with InGaP cladding layers were studied by photoreflectance at various temperatures. The excitonic interband transitions of the InGaAsN/GaAsN/GaAs QW systems were observed in the spectral range above $h \nu=E_{\mathrm{g}}(\mathrm{InGaAsN})$. The confinement potential of the system with strain compensating GaAsN barriers became one step broader, thus more quantum states and larger optical transition rate were observed. A matrix transfer algorithm was used to calculate the subband energies numerically. Band gap energies, effective masses were adopted from the band anti-crossing model with band-offset values adjusted to obtain the subband energies to best fit the observed optical transition features. A spectral feature below and near the GaAs band gap energy from GaAs barriers is enhanced by the GaAs/InGaP interface space charge accumulation induced internal field.
\end{abstract}

(C) 2005 Elsevier Ltd. All rights reserved.

Keywords: A. Semiconductors; A. Quantum wells; D. Optical properties

\section{Introduction}

Small amount of nitrogen content has profound effect reducing the band gap of nitrogen containing III-V alloys like GaAsN and InGaAsN, which have drawn considerable attention recently because of their interesting physical properties and a wide range of possible optoelectronic applications [1-3]. Following the demonstration of laser diodes based on InGaAsN quantum well (QW) structures, many more imaginative band gap and strain engineering designs are proposed. Considerable flexibility is achieved through the ability of In and $\mathrm{N}$ to not only reduce the band gap of GaAs but also have opposite effect on the lattice constant. As a matter of fact, adding nitrogen into the compressively strained $\mathrm{InGaAs} / \mathrm{GaAs}$ QW can reduce the strain, lower the band gap, and increase the conduction band offset. However, with increasing $\mathrm{N}$ mole fraction, the optical quality of the material may be deteriorated significantly, resulting in a higher threshold current density. For better performance of InGaAsN/GaAs QW lasers, the nitrogen composition of InGaAsN should be very small, and

\footnotetext{
* Corresponding author. Address: Department of Physics, NTNU, 88 Sec. 4 Ting-Chou Road, Taipei 116, Taiwan, ROC. Tel.: + 8862 29346620x133; fax: +886229326408.

E-mail address: lupond@phy03.phy.ntnu.edu.tw (C.R. Lu).
}

0022-3697/\$ - see front matter (C 2005 Elsevier Ltd. All rights reserved. doi:10.1016/j.jpcs.2005.09.059 this will lead to strain increasing for the adequate wavelength $[4,5]$. Inserting strain compensating layers to the InGaAsN/ GaAs QW structure can extend the emission wavelength and reduce the average strain of the system [6,7]. However the effect on optical properties of InGaAsN/GaAs QW by inserting strain compensating GaAsN layers is not well understood, and further experimental studies are needed.

In this paper, optical properties of InGaAsN/GaAsN/GaAs quantum well $(\mathrm{QW})$ structures with $\mathrm{InGaP}$ cladding layers were studied by photoreflectance (PR) spectroscopy at various temperatures, and verified by the photoluminescence (PL), reflectance and transmittance spectroscopies. The excitonic interband transitions of the InGaAsN/GaAsN/GaAs QW systems were observed in the spectral range above $h \nu=E_{\mathrm{g}}$ (InGaAsN). The confinement potential of the system with strain compensating GaAsN barriers is lowered and broadened, therefore more quantum states and larger optical transition rate were observed. A transition feature below the gap of $\mathrm{GaAs}$ barrier corresponds to the optical transition involving confined states in the potential well which is formed by the accumulation space charge induced internal electric fields near the $\mathrm{GaAs} / \mathrm{InGaP}$ interface [8].

\section{Experiment}

The MBE grown InGaAsN/GaAs QW structures consist of an $\operatorname{In}_{0.3} \mathrm{Ga}_{0.7} \mathrm{As}_{0.972} \mathrm{~N}_{0.028}$ well of $6 \mathrm{~nm}$, two GaAs barriers of 
$120 \mathrm{~nm}$ and two $\mathrm{In}_{0.49} \mathrm{Ga}_{0.51} \mathrm{P}$ cladding layers of $100 \mathrm{~nm}$. For the comparative system, $2.5 \mathrm{~nm}$ of the GaAs barriers next to the InGaAsN well were replaced by strain compensating $\mathrm{GaAs}_{0.965} \mathrm{~N}_{0.035}$ layers. Samples were annealed at $700{ }^{\circ} \mathrm{C}$ for twenty minutes under $\mathrm{N}_{2}$ purge. In the PR experiment, an Argon ion laser as the excitation source provided pumping photons, and they generate free electron hole pairs to neutralize the space charge in the system, and thus modulate the internal field strength. The probe light was provided by a quartz halogen lamp and the wavelength was selected by the quarter meter monochromator. Using the phase lock-in technique, the electro-modulated optical responses of the excitonic transition between the quantum well confined hole states and electron states are enhanced. The band edge transition exhibits FranzKeldysh oscillatory (FKO) $[9,10]$ features whose period indicates the strength of the internal field.

\section{Results and discussion}

The PR spectra of the InGaAsN/GaAs QW with and without strain compensating GaAsN barriers at $300 \mathrm{~K}$ are compared in Fig. 1. Pumping photons induced modulation of the internal electric field will result two types of electro-modulated line shape. When the internal electric field is strong, the band edge transition will exhibit FKO features above the band gap energy $[9,10]$. The oscillating features in the spectral range $h \nu>E_{\mathrm{g}}(\mathrm{GaAs})$ of Fig. 1 are originated from the surface field region of the GaAs cap. When the internal electric field is

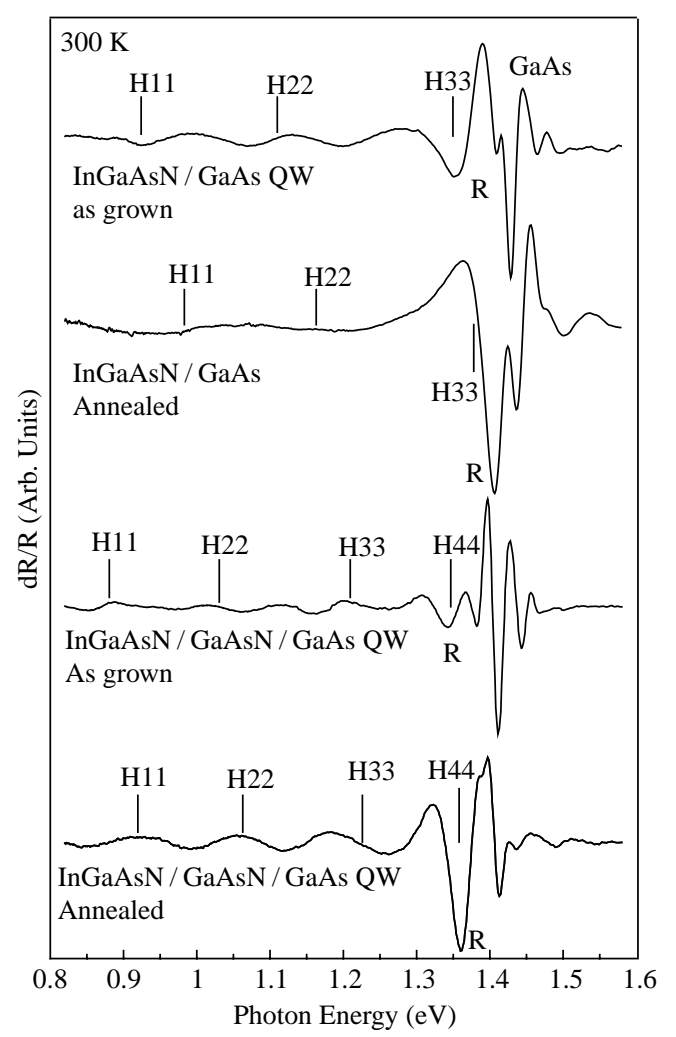

Fig. 1. PR spectra at $300 \mathrm{~K}$ of the InGaAsN/GaAs QW with and without strain compensating GaAsN barriers. Vertical bars and Hnn indicate expected transitions involving the nth heavy hole and electron subbands. weak, the modulated optical responses will be the derivative like line shape $[11,12]$. When photon energy is below and near the band gap of the GaAs, there is a near edge resonance feature labeled $\mathrm{R}$ in the spectra of Fig. 1.

Due to the quantum confinement enhancement, the excitonic interband transitions of the InGaAsN/GaAs QW systems are clearly resolved in the spectral range of smaller energy. Vertical bars in the figure indicate where QW transitions are expected. The annealing process improved the crystal quality and enhanced the optical transition features. Except for the GaAs band edge transition features near $1.42 \mathrm{eV}$, all spectral features blue shifted after annealing. Since, the confinement potential of the system with strain compensating GaAsN barriers is wider and lower, not only more quantum states and thus more spectral features are observed, spectral features are also red shifted. The InGaAsN/GaAs QW subband energies were calculated numerically by using the transfer matrix technique for analyzing multi-layer heterostructures formulated by Vassell et al. [13]. The band gap, electron effective mass, and the bandoffset value were adjusted to obtain the InGaAsN/GaAs QW subband energies to fit the observed optical transition features. Theoretical studies have suggested that the interaction between the nitrogen energy level and the conduction band edge of InGaAs accounts for the compositional dependence of the electronic parameters [14-16]. The nitrogen content dependent band gap value and electron effective mass from the BAC model $[17,18]$ were adopted in the calculation. Interband transitions involving the quantum-confined states of the InGaAsN/GaAs QW with and without strain compensating GaAsN barriers at $300 \mathrm{~K}$ are label in Fig. 1. The symbols H11 indicate the optical transitions involving the ground states of electron and heavy hole, and $\mathrm{H} 22$ indicate those involving the first excited states. There are three electronic states confined in the InGaAsN/GaAs QW and four electronic states confined in the QW system with GaAsN strain compensating layer. Fig. 2

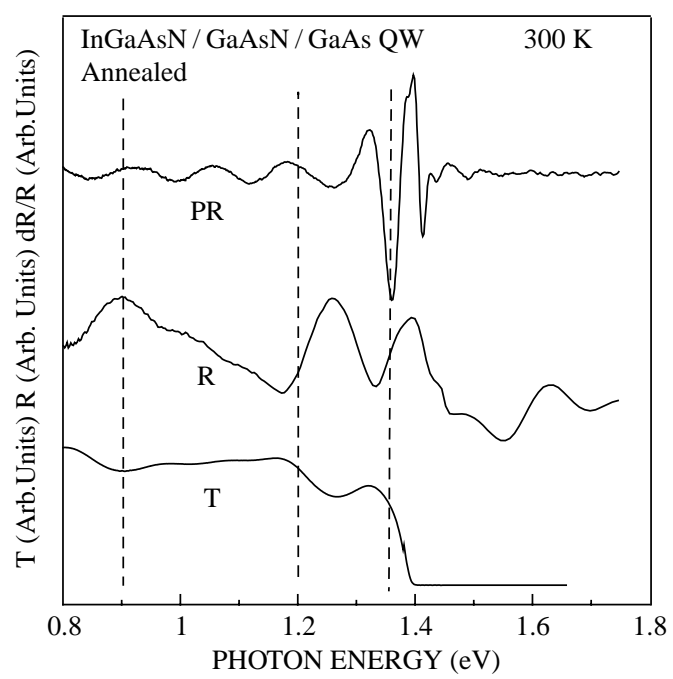

Fig. 2. Comparison of the PR spectra with reflectance and transmittance spectra of the annealed InGaAsN/GaAs QW with strain compensating GaAsN barriers at $300 \mathrm{~K}$. Dashed lines indicate that major transition features agree well. 


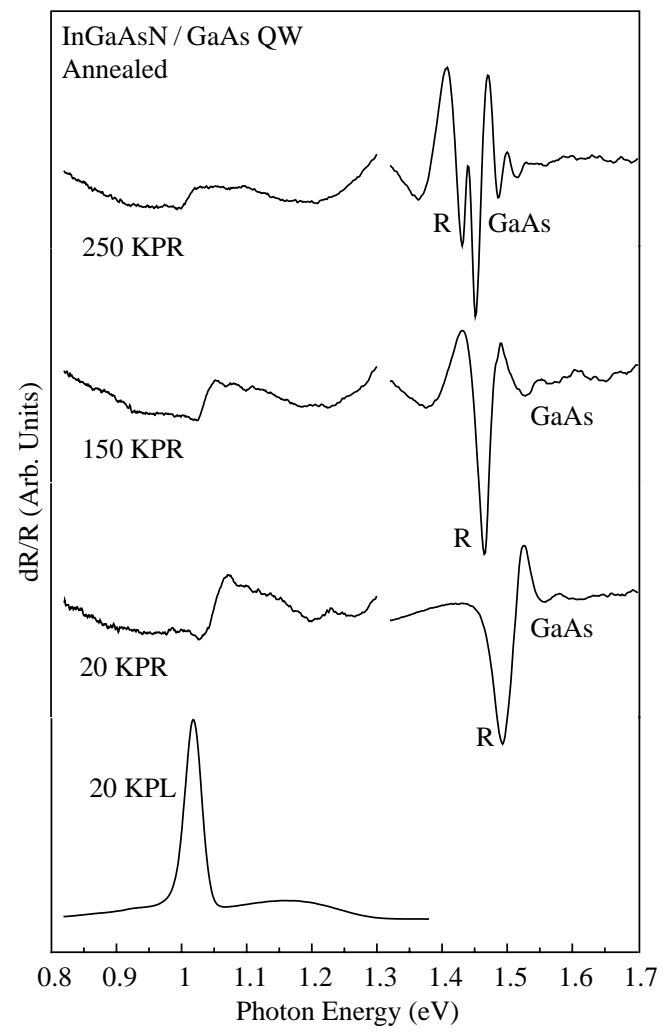

Fig. 3. Temperature dependence of the InGaAsN/GaAs QW PR spectra, and a comparison of PR and PL spectra. The low energy portion of the PR spectrum is magnified for clarity. The PL transition energy is smaller than the PR transition energy.

shows that major optical transition features are verified by the reflectance and transmittance spectra, and more QW transition details are observed in the PR spectrum. Fig. 3 summarizes the temperature dependence of the annealed InGaAsN/GaAs QW and compares with the PL spectra. The QW transition energies observed in the PL spectra are smaller than those observed in the PR spectra. Nitrogen centers in the InGaAsN perturb the conduction band and cause potential fluctuations. The PR experiment is an excitation spectroscopy, and the average band to band transition is observed. The PL originates from the recombination emission, and records the transition of the potential fluctuation edge. Therefore, the PL spectra show smaller transition peak energies. The PR spectral features from InGaNAs/GaAs QW in Fig. 3 are red shifted with band edge as temperature increases. The oscillatory features above the gap of GaAs are caused by the pumping photons induced modulation of the internal electric field in the GaAs surface band bending region. The periods of the FKO features decrease as the temperature decreases. The internal electric field decreases as the temperature is decreased because lowering the temperature tends to freeze out carriers, neutralize the space charge, and thus reduce the internal electric field. As the temperature decreased further, the internal electric field become too small to induce FKO, the GaAs PR feature become a weaker third derivative line shape [9-12], and eventually become irresolvable at very low temperature as show in Fig. 3. The internal field induced by the space charge accumulation near the GaAs/InGaP interfaces [8] will lower the potential in the GaAs barrier and confined electrons near the interface. The confined electron states with energies below the gap of GaAs may be in resonance with the highest electron subband in the InGaAsN/GaAs QW, and increase the optical transition rate. Due to the space charge accumulation near the $\mathrm{GaAs} / \mathrm{InGaP}$ interfaces, the electro-modulation is more efficient in the GaAs barriers and enhances the near edge PR features.

\section{Summary}

We have investigated InGaAsN /GaAs and InGaAsN/ GaAsN/GaAs quantum well (QW) structures with InGaP cladding layers by photoreflectance $(\mathrm{PR})$ at various temperatures. Spectral features are also verified by photoluminescence, reflectance and transmittance spectroscopies. The excitonic interband transitions of the InGaAsN/GaAsN/GaAs QW systems were observed in the spectral range above $h \nu=E_{\mathrm{g}}$ (InGaAsN). The confinement potential of the system with strain compensating GaAsN barriers is one step broader; therefore, more quantum states and larger optical transition rate were observed. A matrix transfer algorithm was used calculate the QW subband energies numerically. Band gap energies and effective masses were adopted from the band anti-crossing model with band off set values adjusted to obtain the subband energies to best fit the observed optical transition features. The PR spectral feature below and near the GaAs band gap energy from the potential induced by the internal field near the $\mathrm{GaAs} / \mathrm{InGaP}$ interface is enhanced by the electro-modulation.

\section{Acknowledgements}

Financial support from National Science Council (NSC 90-2112-M-003-026) and Taiwan Normal University is gratefully acknowledged.

\section{References}

[1] M. Hetterich, M.D. Dawson, A.Yu. Egorov, D. Bernklau, H. Riechert, Electronic states and band alignment in GalnNAs/GaAs quantum-well structures with low nitrogen content, Appl. Phys. Lett. 76 (2000) 1030.

[2] A. Lindsay, E.P. O'Reilly, Theory of enhanced bandgap non-parabolicity in $\mathrm{GaN}_{x} \mathrm{As}_{1-x}$ and related alloys, Solid State Commun. 112 (1999) 443.

[3] C. Ellmers, F. Hohnsdorf, J. Hoch, C. Agert, S. Leu, D. Karaiskaj, M. Hoffman, W. Stolz, W.W. Ruhle, Ultrafast (GaIn)(NAs)/GaAs vertical-cavity surface-emitting laser for the $1.3 \mu \mathrm{m}$ wavelength regime, Appl. Phys. Lett. 74 (1999) 2271.

[4] W. Li, J. Turpeinen, P. Melanen, P. Savolainen, P. Uusimaa, M. Pessa, Effects of rapid thermal annealing on strain-compensated GaInNAs/GaAsP quantum well structures and lasers, Appl. Phys. Lett. 78 (2001) 91.

[5] L.F. Bian, D.S. Jian, S.L. Lu, J.S. Huang, K. Chang, L.H. Li, J.C. Harmand, The effect of inserting strain-compendensated GaAsN layers on the luminescence properties of GaInNAs/GaAs quantum well, J. Cryst. Growth 250 (2003) 339.

[6] W. Li, T. Jouhti, C.S. Peng, J. Konttinen, P. Laukkanen, E.-M. Pavelescu, M. Dumitrescu, M. Pessa, Low-threshold-current 1.32- $\mu \mathrm{m}$ GaInNAs/ GaAs single-quantum-well lasers grown by molecular-beam epitaxy, Appl. Phys. Lett. 79 (2001) 3386. 
[7] E. Tournié, M.-A. Pinault, A. Guzmán, Mechanisms affecting the photoluminescence spectra of GaInNAs after post-growth annealing, Appl. Phys. Lett. 80 (2002) 4148.

[8] P. Krispin, A. Knauer, S. Gramlich, Electric field-induced redistribution of free carriers at isotype (In, Ga)P/GaAs interfaces, Mater. Sci. Eng. B88 (2002) 129.

[9] D.E. Aspnes, A.A. Studna, Schottky-barrier electroreflectance: application to GaAs, Phys. Rev. B 7 (1973) 4605.

[10] R.N. Bhattacharys, H. Shen, P. Parayanthal, F.H. Pollak, T. Coutts, $\mathrm{H}$. Aharoni, Electroreflectance and photoreflectance study of the spacecharge region in semiconductors: ( $\mathrm{In}-\mathrm{Sn}-\mathrm{O}) / \mathrm{InP}$ as a model system, Phys. Rev. B 37 (1988) 4044.

[11] H. Shen, P. Parayanthal, F.H. Pollak, M. Tomkiewicz, T. Drommond, J.N. Schulman, Photoreflectance study of GaAs/AlAs superlattices: fit to electromodulation theory, Appl. Phys. Lett. 48 (1986) 653.

[12] B.V. Shanabrook, O.J. Glembocki, W.T. Beard, Photoreflectance modulation mechanisms in GaAs- $\mathrm{Al}_{x} \mathrm{Ga}_{1-x} \mathrm{As}$ multiple quantum wells, Phys. Rev. B 35 (1987) 2540.
[13] M.O. Vassel, J. Lee, H.F. Lockwood, Multibarrier tunneling in $\mathrm{Ga}_{1-x}$ $\mathrm{Al}_{x} / \mathrm{GaAs}$ heterostructures, J. Appl. Phys. 54 (1983) 5206.

[14] L. Bellaiche, S.H. Wei, A. Zunger, Composition dependence of interband transition intensities in GaPN, GaAsN, and GaPAs alloys, Phys. Rev. B 56 (1997) 10233.

[15] A. Lindsay, E.P. O'Reilly, Influence of nitrogen resonance states on the electronic structure, Solid State Commun. 118 (2001) 313.

[16] C. Skierbiszewski, P. Perlin, P. Wisniewski, W. Knap, T. Suski, W. Walukeiwicz, W. Shan, K.M. Yu, J.W. Ager, E.E. Haller, J.F. Geisz, J.M. Olson, Large, nitrogen-induced increase of the electron effective mass in $\mathrm{In}_{y} \mathrm{Ga}_{1-y} \mathrm{~N}_{x} \mathrm{As}_{1-x}$, Appl. Phys. Lett. 76 (2000) 2409.

[17] W. Shan, W. Walukeiwicz, J.W. Ager III, E.E. Haller, J.F. Geisz, D.J. Friedman, J.M. Olson, S.R. Kurtz, Band anticrossing in GaInNAs alloys, Phys. Rev. Lett. 82 (1999) 1221.

[18] W. Shan, W. Walukiewicz, K.M. Yu, J.W. Ager III, E.E. Haller, J.F. Geisz, D.J. Friedman, J.M. Olson, Sarah.R. Kurtz, Effect of nitrogen on the electronic band structure of group III-N-V alloys, Phys. Rev. B 62 (2000) 4211. 